\title{
Pitfalls of simulation-based machine learning in optoelectronic device design
}

Joachim Piprek

NUSOD Institute, Newark, DE 19714-7204, United States, E-mail: piprek@nusod.org

\begin{abstract}
Numerical simulation and machine learning represent opposite approaches to computational analysis of the real world, deductive vs. inductive. However, both methods suffer from various uncertainties and even their combination often fails to link theory and reality. Focusing on GaN-based light-emitting diode (LED) design optimization, this paper evaluates examples of simulation-based machine learning from a physics point of view. Strategies are suggested for achieving more realistic predictions.
\end{abstract}

Keywords: machine learning, deep learning, neural networks, artificial intelligence, numerical simulation, design optimization, light-emitting diode

Computer simulations embed theoretical models into a practical environment. ${ }^{1}$ This enables a realistic test of such models by comparing calculated results to measurements. Simulations can thereby help explain experimental results that would otherwise be hard to understand. Simulations also allow for performance predictions for novel devices. However, it is well known that initial simulation results hardly ever agree with experimental data. ${ }^{2}$ In other words, computer simulations often fail to represent the real world and create a virtual reality instead in which unreal effects may happen (Fig. 1). This is not surprising as mathematical models always simplify reality. There are different levels of simplification, from short analytical formulas to complex systems of equations, which are all based on specific assumptions about relevant physical processes. Certain assumptions may be inappropriate in a practical situation. What is more, contradicting models may deliver almost identical results. ${ }^{3}$ Another key reason for disagreements is the employment of unrealistic material parameters. Published values of such parameters often vary substantially. ${ }^{4}$ Careful adjustments of computer simulations are required to find agreement between theory and reality. ${ }^{2}$

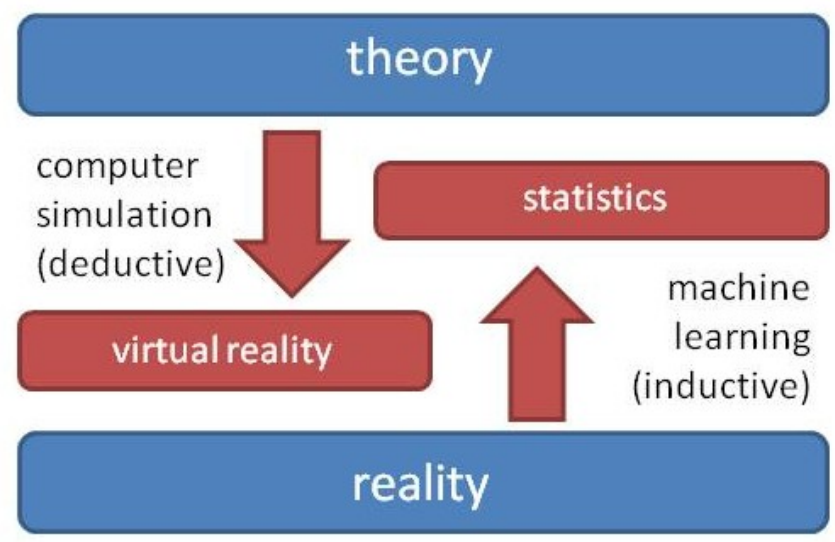

Fig. 1: Deductive vs. inductive method. 
Machine learning, on the other hand, usually collects data in the real world and performs statistical analyses (Fig. 1). This is especially valuable when the amount of data is very large and hard to digest. Deep learning is currently the most popular machine learning method and it is based on multilayered artificial neural networks (ANNs). ${ }^{5}$ Many data sets are needed to train an ANN. Due to scarcity and scatter of real-world data, experimental data collection is often replaced by computer simulations based on established theories. Such physics-based machine learning methods are increasingly utilized in materials science. ${ }^{6}$ Various semiconductor material systems are explored and optimized for applications in optoelectronics. ${ }^{7,8}$ Simulation-based machine learning is also popular with photonic devices, ${ }^{9}$ including solutions to Maxwell's equations that involve only few material parameters. Compared to photonics, optoelectronic devices are much more complex as they combine optical, electronic, and thermal processes. As indicated above, computer simulations of such devices not only involve various modeling approximations in need of validation, but also many uncertain material parameters. However, simulationbased machine learning is employed in the design of optoelectronic devices such as laser diodes, ${ }^{10}$ semiconductor optical amplifiers, ${ }^{11}$ and solar cells. ${ }^{12,13}$ Light-emitting diodes (LEDs) in particular are a common target of machine learning. ${ }^{14,15,16}$

In the following, we evaluate a few of these machine-learning applications that are focused on GaN-based LEDs. These blue light emitters have been receiving great attention in recent years due to their widespread utilization in lighting, displays and other fields. ${ }^{17}$ Countless and partially contradicting GaN-LED simulation studies have been published, with the LED efficiency being in the center of interest in most cases. ${ }^{18}$ Not surprisingly, one of the first machine learning applications to GaN-LEDs is focused on efficiency improvements. ${ }^{19}$ The authors employ a genetic algorithm to select combinations of basic semiconductor layer structures with random material composition, thickness, and doping. Utilizing a Matlab-based LED model, the design is successively improved to maximize an efficiency-related fitness function. After about 500 generations with a typical population size of 1000, an optimum LED design is identified that enhances the efficiency by limiting the electron leakage from the InGaN quantum well (QW) active layers. A further leakage reduction is discovered for a novel design of the electron blocking layer (EBL). The EBL is split up into 10 individual $3 \mathrm{~nm}$-thick layers with different composition. However, this optimization strategy ignores the strong influence of band-offset and $\mathrm{Mg}$ doping reported earlier. $^{20}$

Mg doping optimization is included in a follow-up paper by some of the same authors which now split the EBL into 1nm-thin sub-layers of different compositions and doping densities. ${ }^{21}$ The genetic algorithm identifies an optimum EBL design of 12 different AlGaInN layers with uniform $\mathrm{Mg}$ doping density of $10^{19} \mathrm{~cm}^{-3}$, which is the maximum of the doping range considered, in agreement with earlier findings. ${ }^{20}$ An internal quantum efficiency (IQE) of 0.59 is predicted for the optimized LED design at $200 \mathrm{~A} / \mathrm{cm}^{2}$ current density, which is a significant improvement compared to IQE $=0.43$ with the reference LED design. IQE represents the ratio between the number of photons generated inside the QWs to the number of electrons injected into the device. It thereby accounts for carrier losses caused by non-radiative recombination or leakage. ${ }^{22}$

For evaluation and validation, we simulate the same LED structures employing the widely used APSYS software. ${ }^{23}$ The code self-consistently computes carrier transport, the wurtzite electron band structure of strained QWs, and photon emission. Schrödinger and Poisson equations are solved iteratively in order to account for the QW deformation with changing device bias (quantum-confined Stark effect). 
The transport model includes drift and diffusion of electrons and holes, Fermi statistics, built-in polarization and thermionic emission at hetero-interfaces, as well as all relevant radiative and nonradiative recombination mechanisms. In agreement with the reference papers, self-heating is excluded from our study. More details on the employed device models are given elsewhere. ${ }^{24}$

\begin{tabular}{|c|c|c|c|}
\hline Layer & Composition & Doping & Thickness \\
\hline p-cladding & GaN:Mg & $1 \times 10^{19} \mathrm{~cm}^{-3}$ & $200 \mathrm{~nm}$ \\
\hline p-EBL & $\mathrm{Al}_{0.19} \mathrm{Ga}_{0.81} \mathrm{~N}: \mathrm{Mg}$ & $1 \times 10^{19} \mathrm{~cm}^{-3}$ & $20 \mathrm{~nm}$ \\
\hline p-injector & GaN:Mg & $1 \times 10^{19} \mathrm{~cm}^{-3}$ & $4 \mathrm{~nm}$ \\
\hline spacer & $\mathrm{GaN}$ & & $6 \mathrm{~nm}$ \\
\hline quantum well & $\mathrm{In}_{0.15} \mathrm{Ga}_{0.85} \mathrm{~N}$ & & $3 \mathrm{~nm}$ \\
\hline n-barrier & GaN:Si & $5 \times 10^{18} \mathrm{~cm}^{-3}$ & $10 \mathrm{~nm}$ \\
\hline quantum well & $\mathrm{In}_{0.15} \mathrm{Ga}_{0.85} \mathrm{~N}$ & & $3 \mathrm{~nm}$ \\
\hline n-barrier & GaN:Si & $5 \times 10^{18} \mathrm{~cm}^{-3}$ & $10 \mathrm{~nm}$ \\
\hline quantum well & $\mathrm{In}_{0.15} \mathrm{Ga}_{0.85} \mathrm{~N}$ & & $3 \mathrm{~nm}$ \\
\hline n-barrier & GaN:Si & $5 \times 10^{18} \mathrm{~cm}^{-3}$ & $10 \mathrm{~nm}$ \\
\hline quantum well & $\mathrm{In}_{0.15} \mathrm{Ga}_{0.85} \mathrm{~N}$ & & $3 \mathrm{~nm}$ \\
\hline n-barrier & GaN:Si & $5 \times 10^{18} \mathrm{~cm}^{-3}$ & $10 \mathrm{~nm}$ \\
\hline quantum well & $\mathrm{In}_{0.15} \mathrm{Ga}_{0.85} \mathrm{~N}$ & & $3 \mathrm{~nm}$ \\
\hline n-cladding & GaN:Si & $7 \times 10^{18} \mathrm{~cm}^{-3}$ & $200 \mathrm{~nm}$ \\
\hline
\end{tabular}

Tab. 1: Layer structure of the reference LED (EBL - electron blocking layer). ${ }^{21}$

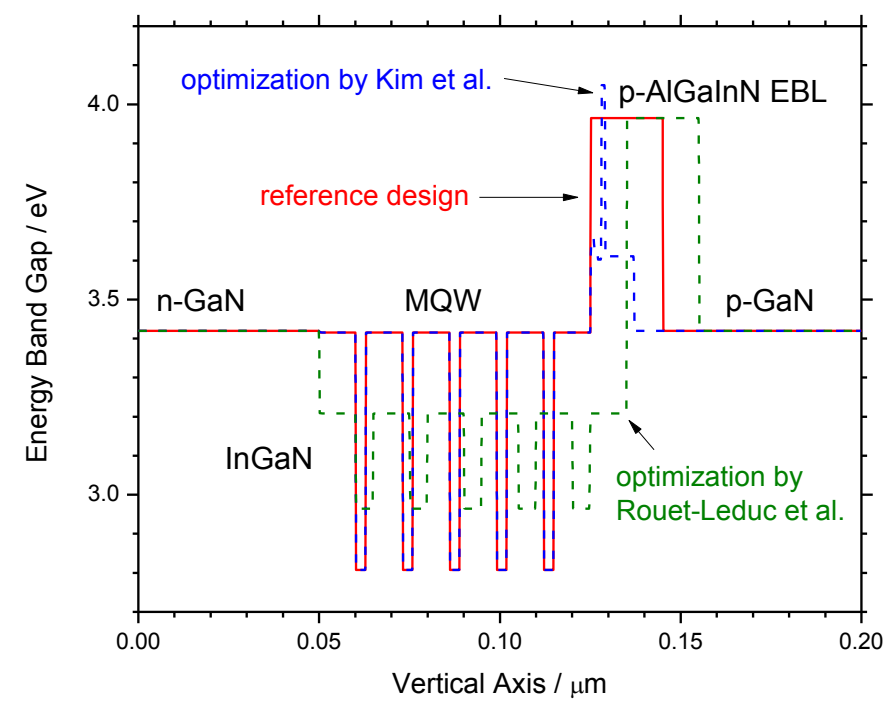

Fig. 1: Comparison of LED band gap profiles (MQW - multiple quantum well, EBL - electron blocking layer).

The layer structure of the reference LED is given in Table $1 .^{21}$ The vertical band gap profiles are plotted in Fig. 1 for the reference LED (red line) and two optimized designs. The calculated LED efficiency is known to strongly depend on material parameters such as Auger recombination coefficient, ${ }^{25}$ 
built-in polarization, ${ }^{26}$ band offset at hetero-junctions, ${ }^{26}$ and hole mobility, none of which is specified in the cited paper. ${ }^{21}$ In order to reproduce the reference IQE characteristic, we here assume an Auger coefficient of $\mathrm{C}=10^{-30} \mathrm{~cm}^{6} / \mathrm{s}$ as well as a conduction band offset ratio of 0.6 and a polarization screening factor of 0.5 , all within the range of published values. However, the hole mobility is seldom mentioned. Lower hole mobility leads to enhanced electron leakage into the Mg-doped layers, thereby reducing the efficiency. The solid lines in Fig. 2 demonstrate the influence of the hole mobility on the simulated IQE characteristic. A typical hole mobility of $1 \mathrm{~cm}^{2} / \mathrm{Vs}$ produces about $1 \%$ electron leakage at $200 \mathrm{~A} / \mathrm{cm}^{2}$ current density (blue line in Fig. 2). A 10-times lower hole mobility gives $25 \%$ leakage and it moves our results close to the IQE reported in the reference paper (red line in Fig. 2). ${ }^{21}$ The optimized LED structure promises improved electron blocking, as suggested by the blue bandgap profile in Fig. 1. Indeed, this LED design produces a somewhat higher efficiency by reducing the electron leakage to $19 \%$ (red dashed line in Fig. 2). But we are unable to confirm the complete leakage elimination, or the strong efficiency improvement predicted in the reference paper, even after testing other parameter combinations. Likely reasons are differences between other material parameters and/or between the underlying models. As illustrated in Fig. 2, modeling uncertainties produce a substantial error range in the simulation, which is larger than the efficiency improvement achieved by the design optimization at $200 \mathrm{~A} / \mathrm{cm}^{2}$. Simulation models and parameters should be first calibrated by comparison to relevant measurements of the reference device. $^{2}$ This is often a time-consuming process but it is a prerequisite for reliable performance predictions.

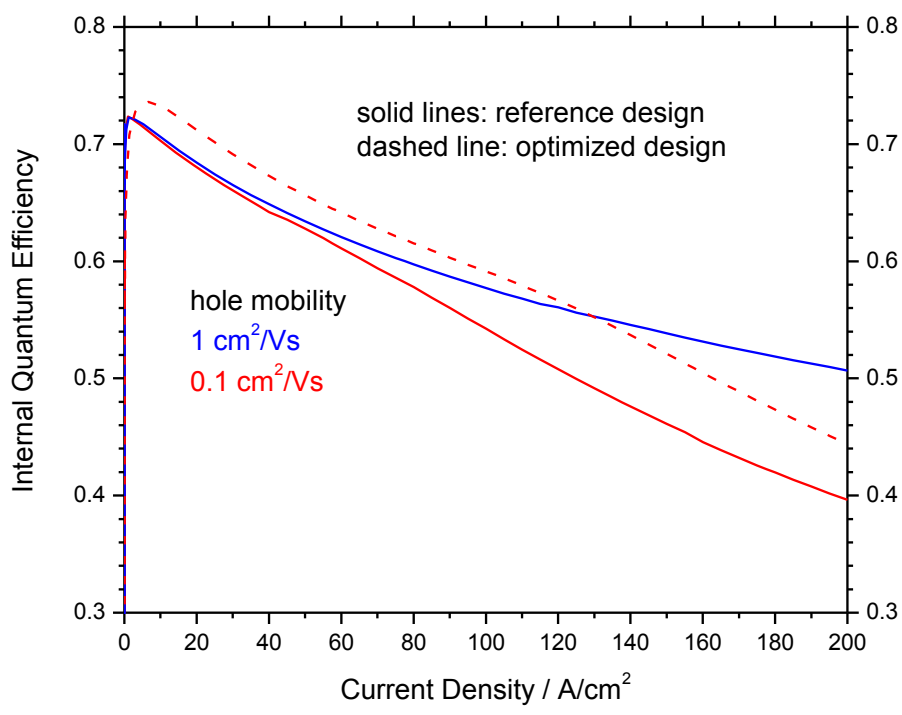

Fig. 2: LED efficiency simulation for designs by Kim et al. ${ }^{21}$

However, such continuum models ignore the atomistic structure of very thin layers. The optimized LED structure features a $1 \mathrm{~nm}$ thick AlGaInN layer with only $5 \%$ Indium content inside the EBL (blue dashed line in Fig. 1), ${ }^{21}$ for which the average lateral distance of Indium atoms is substantially larger than the layer thickness. In other words, the assumption of a uniform energy barrier is incorrect. What is more, such thin barrier enables carrier tunneling which is not considered in these simulations. Thus, the machine learning results clearly overstep the validity limits of the underlying model. Design space boundaries should be carefully evaluated and strictly observed. 
The above GaN-LED design optimization is based on the popular assumption that electron leakage is the main reason for the efficiency reduction with rising current. However, other authors blame QW Auger recombination for this efficiency droop, which can be equally validated by LED simulations, even for the same device structure. ${ }^{3}$ Both carrier loss mechanisms can be reduced by lowering the QW carrier density. Rouet-Leduc et al. published such GaN-LED design optimization based on active machine learning, ${ }^{14}$ but without revealing much detail about LED parameters. Utilizing the APSYS software, the authors successively perform about 1000 simulations by varying the composition of their five InGaN quantum wells independently. The QW barrier composition is also changed and the QW thickness is adjusted to maintain a nearly constant emission wavelength. This strategy eventually leads to an IQE improvement from 0.55 to 0.78 at $75 \mathrm{~A} / \mathrm{cm}^{2}$ current density. Their machine learning algorithm finds the highest efficiency for widened QWs with about 11\% Indium sandwiched between InGaN barriers with $5 \%$ Indium. ${ }^{14}$ This design optimization is quite intuitive as thicker QWs reduce the QW carrier density and shallower QWs shrink the built-in polarization field that separates electrons and holes inside the QW.

For evaluation, we apply this optimization strategy to the same LED reference structure simulated above (Tab. 1). Our IQE of 0.58 at $75 \mathrm{~A} / \mathrm{cm}^{2}$ (Fig. 3) is close to the value 0.55 reported by Rouet-Leduc et al. for their reference LED. The proposed addition of 5\% Indium to the QW barriers results in the dashdot line in Fig. 3. The efficiency at $75 \mathrm{~A} / \mathrm{cm}^{2}$ drops due to enhanced electron leakage enabled by the lower QW barriers. Widening the QW from $3 \mathrm{~nm}$ to $5 \mathrm{~nm}$ causes a rising efficiency as the QW carrier density is reduced (dash-dot-dot line in Fig. 3). The final reduction of the QW Indium content to $11 \%$ lowers the efficiency again due to stronger leakage from shallow QWs, despite a weaker polarization (dashed line in Fig. 3). The resulting band gap profile is shown as green line in Fig. 1. However, at the target current density of $75 \mathrm{~A} / \mathrm{cm}^{2}$, the IQE always remains lower than with the reference LED. Obviously, the success of this design optimization depends on more parameters than those considered by the authors. They skipped an essential step, namely the identification of the dominating loss mechanism in their simulation, which is supposed to be suppressed in the optimized design. Simulations should always investigate internal device physics first before developing design optimization strategies.

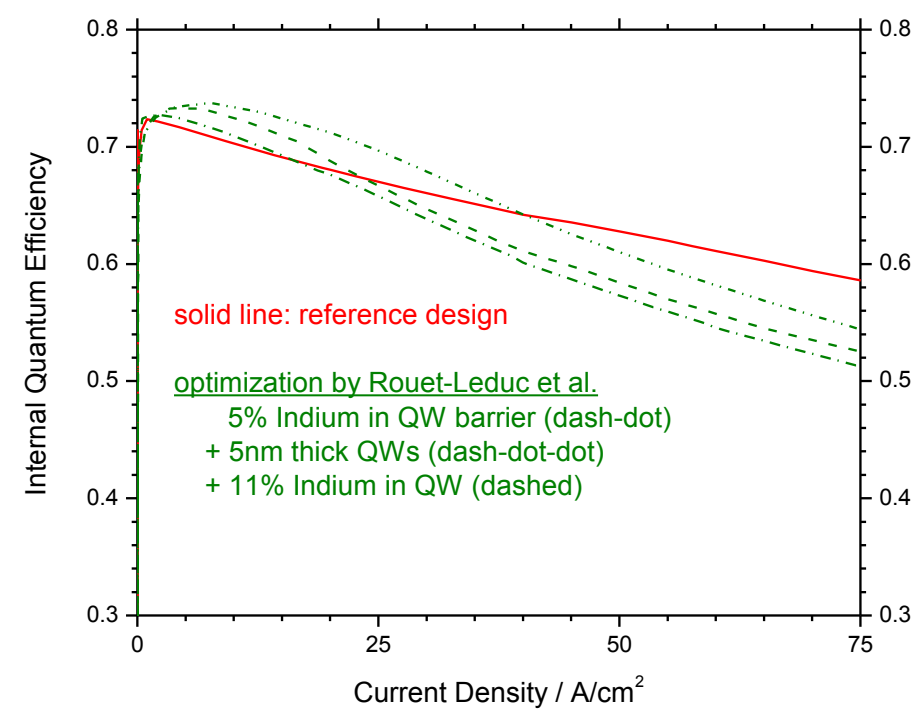

Fig. 3: LED efficiency simulation of the design changes proposed by Rouet-Leduc et al. ${ }^{14}$ 
In his Ph.D. thesis, ${ }^{27}$ Rouet-Leduc applied similar machine learning methods to the fitting of simulated efficiency characteristics to a measured one. This approach accounts for well-known uncertainties of key material parameters: built-in polarization, recombination coefficients, QW carrier scattering time, and the exchange coefficient controlling the band-gap shrinkage with rising QW carrier density. Based on a large set of known simulation results for random material parameters, active machine learning is used to select material parameter sets for new simulations, thereby successively increasing the available data base. Reasonable agreement between measurement and simulation is achieved after about 300 iterations. While this is an important and valuable approach, there are two main shortcomings. First, the final fit parameters are not listed and discussed, i.e., this machine learning demonstration does not improve our understanding of device physics. Second, the time-consuming generation of hundreds of APSYS simulation results needs to start over for any design modification. A fitting procedure based on physical insight is probably faster and certainly more educating.

These examples demonstrate that simulation-based machine learning is extremely challenging due to the complexity of optoelectronics device physics. Reliable performance predictions are impossible without thorough validation of simulation models, including material parameters, and strict observance of design space limits. We here focus on problems related to LED physics, other machine learning pitfalls are investigated elsewhere. ${ }^{28,29,30,31,32,33}$ What is more, new scientific discoveries are unlikely as machine learning usually interpolates between known numerical results. This dilemma may be addressed in various ways, some of which are outlined in the following. The key element of such approaches is the involvement of experimental data.

Material parameters are a main source of device simulation uncertainties. Big-data materials science could provide valuable input. For instance, the Materials Genome Initiative demonstrates a synergetic combination of theoretical models, computational methods, and experimental procedures. ${ }^{34,35}$ Another example is the FAIR Data Initiative. ${ }^{36}$ Such activities hopefully lead to more reliable models for key material properties relevant to optoelectronic device simulations. High-end models for sophisticated properties such as optical gain and absorption could be calculated separately and then imported into the full device simulation via ANN or other suitable means. ${ }^{37}$ Parameter uncertainties should be considered in the simulation to enable a much needed establishment of error ranges for calculated results. ${ }^{38}$

The initial disagreement between simulation results and performance measurements is often eliminated by adjusting parameters in the model. ${ }^{2}$ Each parameter usually requires a different measurement for calibration. The industrial fabrication of optoelectronic devices often includes the automatic acquisition of performance data from various measurements. Some values scatter significantly, which may be related to process variations. Machine learning from measured data can be used to analyze such fluctuations and to select the experimental characteristics most appropriate for evaluating simulations.

Assumptions about the simulated device design are usually based on technological intentions and not on the actual structure fabricated, hindering a comparison between simulation and experiment. Typical examples are layer thickness, composition, and doping profile. Direct measurements of such structural data should be preferred in the simulation setup, whenever possible.

In conclusion, the combination of inductive and deductive computational methods typically suffers from a deficient inclusion of experimental data. Theoretical and experimental research should be documented and analyzed in much more detail to make results reproducible and actionable. 


\section{REFERENCES}

${ }^{1}$ J. Piprek (ed.), Handbook of optoelectronic device modeling and simulation, CRC Press, 2017

${ }^{2}$ J. Piprek et al., J. Quantum Electron., 38 (2002) 1253; http://dx.doi.org/10.1109/JQE.2002.802441

${ }^{3}$ J. Piprek, Appl. Phys. Lett. 107 (2015) 031101 ; http://dx.doi.org/10.1063/1.4927202

${ }^{4}$ J. Piprek et al., Appl. Phys. Lett. 106 (2015) 101101; https://doi.org/10.1063/1.4914833

${ }^{5}$ Y. LeCunn et al., Nature 531 (2015) 436; https://doi.org/10.1038/nature14539

${ }^{6}$ J. Schmidt et al., Comp. Mat. 5 (2019) 83; https://doi.org/10.1038/s41524-019-0221-0

${ }^{7}$ Z. Liu et al., InfoMat 2 (2020) ; https://doi.org/10.1002/inf2.12099

${ }^{8}$ Q. Lu et al.; https://arxiv.org/abs/1910.13551

${ }^{9}$ S. Molesky et al., Nature Phot. 12 (2018) 659; https://arxiv.org/abs/1801.06715v1

${ }^{10}$ Z. Ma et al., SPIE Proc. 11209 (2019) 112092X; https://doi.org/ 10.1117/12.2548701

${ }^{11}$ F. Hakimian et al., Opt. Quant. Electr. 52 (2020) 48; https://doi.org/10.1007/s11082-019-2174-4

${ }^{12}$ R. Galphade, J. Electr. Pow. Ener. Sys. 3 (2017) 14; https://doi.org/10.11648/j.ijecec.20170301.12

${ }^{13}$ S. Cicic and S. Tomic, Solar Energy Materials and Solar Cells 200 (2019) 109878; https://doi.org/10.1016/j.solmat.2019.03.050

${ }^{14}$ B. Rouet-Leduc et al., Sci. Rep. 6 (2016) 24862; https://doi.org/10.1038/srep24862

${ }^{15}$ M. A. B. Janai et al., Organ. Electron. 63 (2018) 257; https://doi.org/10.1016/j.orgel.2018.09.029

16 J. Fan et al., Photon. J. 12 (2020) 8200218; https://doi.org/10.1109/JPHOT.2019.2962818

${ }^{17}$ H. S. Wasisto et al., Appl. Phys. Rev. 6 (2019) 041315; https://doi.org/10.1063/1.5096322

${ }^{18}$ G. Verzellesi et al., Appl. Phys. Rev. 114 (2013) 071101; https://doi.org/10.1063/1.4816434

${ }^{19}$ D. Zhu et al., Appl. Phys. Expr. 5 (2012) 012102; https://doi.org/10.1143/APEX.5.012102

${ }^{20}$ J. Piprek et al., Opt. Quant. Electron. 42 (2010) 89-95; https://doi.org/10.1007/s11082-011-9437-z

${ }^{21}$ D. Y. Kim et al., IEEE Photonics J. 7 (2015) 1300209; https://doi.org/10.1109/JPHOT.2014.2387263

${ }^{22}$ J. Piprek, Phys. Status Solidi A 207 (2010) 2217; https://doi.org/10.1002/pssa.201026149

${ }^{23}$ Crosslight Software Inc., Canada (http://www.crosslight.com).

${ }^{24}$ J. Piprek, Ch. 9 in: Semiconductor Optoelectronic Devices: Introduction to Physics and Simulation, Academic Press, San Diego, 2003.

${ }^{25}$ J. Piprek, F. Römer, and B. Witzigmann, Appl. Phys. Lett. 106 (2015) 101101; https://doi.org/10.1063/1.4914833

${ }^{26}$ J. Piprek and Z. M. Li, Appl. Phys. Lett. 102 (2013) 131103; http://dx.doi.org/10.1063/1.4799672

${ }^{27}$ B. Rouet-Leduc, "Machine learning for materials science." Ph.D. Thesis, Univ. of Cambridge , 2017, Ch. 5

${ }^{28}$ Z. C. Lipton et al.; https://arxiv.org/abs/1807.03341

${ }^{29}$ P. Riley, Nature 572 (2019) 27; https://doi.org/10.1038/d41586-019-02307-y

${ }^{30}$ B. Kailkhura et al., Comp. Mat. 5 (2019) 108; https://doi.org/10.1038/s41524-019-0248-2

${ }^{31}$ A. Zunger, Nature 566 (2019) 447; https://doi.org/10.1038/d41586-019-00676-y

${ }^{32}$ S. Chugh et al., J. Lightw. Technol. 37 (2019) 6080; https://doi.org/ 10.1109/JLT.2019.2946572

${ }^{33}$ D. Heaven, Nature 574 (2019) 163; https://doi.org/10.1038/d41586-019-03013-5

${ }^{34}$ J. J. de Pablo et al., Comp. Mat. 5 (2019) 41; https://doi.org/10.1038/s41524-019-0173-4

${ }^{35}$ T. Lookman et al., Comp. Mat. 5 (2019) 21; https://doi.org/10.1038/s41524-019-0153-8

${ }^{36}$ C. Draxl and M. Scheffler, J. Phys.: Mater. 2 (2019) 036001, https://doi.org/10.1088/2515-7639/ab13bb

${ }^{37}$ J. Piprek et al., SPIE Proc. 5729 (2005) 170; https://doi.org/10.1117/12.584570

${ }^{38}$ J. Piprek, Opt. Quant. Electr. 51 (2019) 60; https://doi.org/10.1007/s11082-019-1776-1 